\title{
Synovial sarcoma of the floor of the mouth: a rare case report
}

\author{
Yannan Wang ${ }^{\dagger}$, Feiya Zhu ${ }^{\dagger}$ and Kai Wang ${ }^{*}$
}

\begin{abstract}
Background: Head and neck Synovial sarcoma (SS) accounts for 3-10\% of all total body SS. It is rare to find it in the oral cavity, especially on the floor of the mouth.

Case presentation: We present a 44-year-old Chinese male, who had been misdiagnosed as fibroadenoma, with a swelling on the right submandibular region for more than 3 months. The radiology examinations and the pathology results indicate the diagnosis of SS of the floor of the mouth.

The patient only had a surgical operation, without radiotherapy and chemotherapy. At the first follow-up, the patient exhibited no clinical or radiographic complications, and the patient was asymptomatic on subsequent visits.

Conclusions: Misdiagnosis results the delay of diagnosis and treatment of SS. Immunohistological analysis might be the most important tool to confirm the diagnosis of SS.
\end{abstract}

Keywords: Synovial sarcoma, The floor of the mouth, Diagnosis, Surgical treatment, Case report

\section{Background}

Synovial sarcoma (SS) is a rare malignant neoplasm of unknown histological origin, accounting for $5.6 \% \sim 10 \%$ of all soft tissue sarcomas [1]. It is generally believed as originating from primitive undifferentiated or pluripotent mesenchymal cells [2].. Most studies have found that the age of patients with SS ranged from 15 to 40 years old [3], and approximately $66 \%$ of the patients were male [4].

The tumor usually occurs in close association with tendon sheaths, bursae, and joint capsules, primarily in the para-articular regions of the extremities. Head and neck SS accounts for $3-10 \%$ of all total body SS, with high incidences in the hypopharynx, the postpharyngeal region, and the parapharyngeal space $[5,6]$. It's rare to found it in the oral cavity, especially on the floor of the mouth. As we know, only 31 intraoral cases have been reported and 2 of them were on the floor of the mouth [1]. The diagnosis of oral SS is difficult because of atypical clinical features and obscure location. Here we present a case report of SS that was previously misdiagnosed as fibroadenoma.

\footnotetext{
* Correspondence: 188102160@csu.edu.cn

†Yannan Wang and Feiya Zhu contributed equally to this work.

Department of Oral and Maxillofacial Surgery, The Second Xiangya Hospital of Central South University, Renmin Road, No 139, Changsha 410011, Hunan, China
}

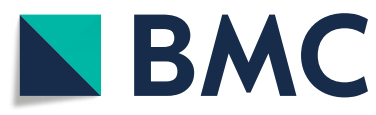

(C) The Author(s). 2020 Open Access This article is distributed under the terms of the Creative Commons Attribution 4.0 International License (http://creativecommons.org/licenses/by/4.0/), which permits unrestricted use, distribution, and reproduction in any medium, provided you give appropriate credit to the original author(s) and the source, provide a link to the Creative Commons license, and indicate if changes were made. The Creative Commons Public Domain Dedication waiver
A 45-year-old male reported that he had a swelling approximately $5.2 \times 2.8 \times 5.9 \mathrm{~cm}$ on the right submandibular region for more than 2 months and was admitted to the local hospital. At the local hospital, the diagnosis of fibroadenoma on the floor of mouth was made. An incisional biopsy was performed under guidance of ultrasound and was submitted for histopathologic examination, the examination revealed that expansion of the lymphatic vessels could be seen in the right sublingual. After an intra-oral excision in the floor of mouth of the right submandibular region (Inferior to the mylohyoid), the immunohistochemistry showed Vimentin was positive, Ki-67 percentage was about 35\%, CD34, S-100, CK, P63 and LCA were negative. After discharged from that hospital, he felt that the swelling had grown again in the same area, even rapidly in the near week.

One month after the first operation at local hospital, the patient was referred to our hospital for a growth nodule on the floor of the mouth, associated with pain, numbness, and dyspnea. Intraoral examination revealed a proliferative and ulcerated mass measuring approximately $6.0 \times 1.0 \mathrm{~cm}$ in the right sublingual involving the right floor of the mouth extending from the alveolus of the left mandibular cuspid to the right mandibular 2nd molar teeth and extending anteriorly crossing the midline of the tongue. The mass was firm, with an unclear \footnotetext{
(http://creativecommons.org/publicdomain/zero/1.0/) applies to the data made available in this article, unless otherwise stated.
} 
border, and the surface covered with black pseudomembranous (Fig. 1). Computed tomography (CT) demonstrated multiple lymph node metastases in the right neck, bilateral submandibular and submental region (Fig. 2a, b), and a mass on right sternocleidomastoid muscle (Fig. 2b, c). Positron Emission TomographyComputed Tomography (PET-CT) also confirmed the CT demonstration. The hematoxylin and eosin (HE)stained section revealed the tumor composed of spindle cells with a higher proportion of nuclei, also with indistinct cytoplasmic borders (Fig. 3). The immunohistochemistry revealed epithelial membrane antigen (EMA), CD99, TLE-1, and Bcl-2 were positive while SMA, CD34, CK, CD68, P63, LCA, and S100 were negative. The above results were consistent with a histopthological diagnosis of SS. The patient developed severe dyspnea and lips cyanosis 1 day before the operation and emergency tracheotomy was performed.

The surgical treatment included primary tumor resection, cervical lymph node dissection, and anterolateral musculocutaneous flap reconstruction (Figs. 4, 5, 6, 7), During the operation, about $0.5 \times 0.5 \times 1 \mathrm{~cm}$ tissue was harvested from the anterior, posterior, internal, external, and basal parts of the surgically removed area. The frozen biopsy results indicated complete excision of the tumor. And 9 days after surgery, the patient was discharged.

Within 1 year after surgery, the patient was seen in our out-patient clinic every month to check the recovery and whether there was recurrence or lymph node metastasis. Six months later, the first radiographic postoperative follow-up showed no recurrence. One year after

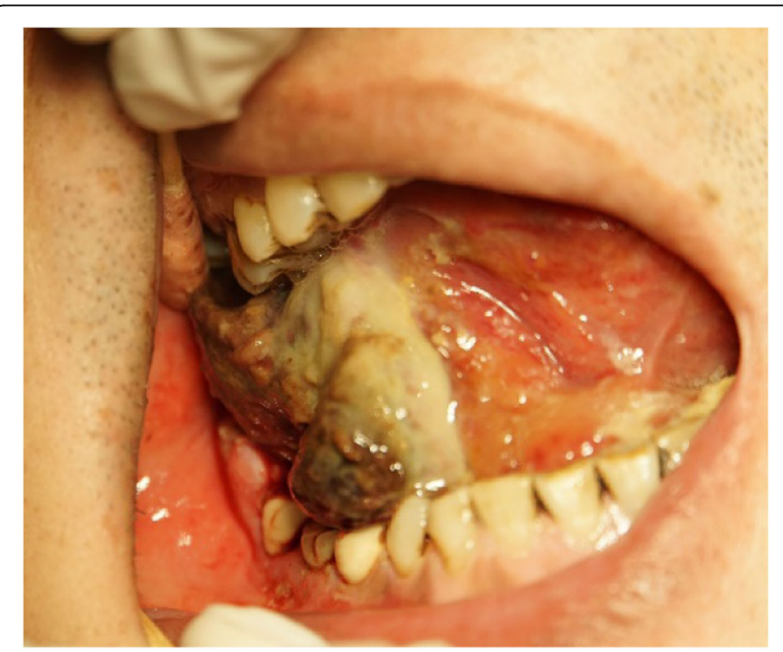

Fig. 1 The tumor was located on the right floor of mouth and ventral part of tongue, with bleeding and necrosis on the surface. Due to poor hygiene conditions in the mouth of the patient, there are food residues, etc., and the surface of the tumor is white. The black part is the tumor tissue of ischemic necrosis. Extra oral examination revealed limited mouth opening that was about $1.5 \mathrm{~cm}$ surgery, PET-CT examination was performed, and the patient had no recurrence and distant metastasis. Then the patient was followed up at six monthly intervals and is still alive with no evidence of recurrence after 46 months since undergoing curative intent surgery.

\section{Discussion and conclusions}

\section{The causes and tissue origin of SS}

Synovial sarcoma (SS) was first documented by Simon in 1865 [7] and was so named in 1934 by Sabrazes et al. [8]. The causes and pathogenesis of SS remain unknown. it is generally accepted that SS is derived from mesenchymal stem cells with multiple differentiation potential, and not from synovial tissue [9]. Nearly 90 to $95 \%$ of SS demonstrates specific $\mathrm{t}(\mathrm{x} ; 18)$ (p11.2-q11.2) chromosomal translocation that forms the SYT-SSX fusion gene [9-11], which promotes synovial sarcoma cells through the Wnt / $\beta$-catenin, PcG, and ERK signaling pathways [12]. Moreover, TGF- $\beta 1$, Smad, Snail, and Slug are also involved in the development of SS through the EMT pathway [13].

\section{Clinical manifestations}

SS usually occurs in the lower limbs and trunk, especially in the periarticular soft tissues [4]. Head and neck SS accounts for $3-10 \%$ of all total body SS [5]. SS lacks typical clinical manifestations, about 50\% of presents with pain, some with dysphagia, dyspnea, hoarseness, headache, limited mouth opening, bleeding and lower lip numbness caused by nerve oppression when occurs in the oral cavity $[14,15]$. It is usually a slow-growing tumor increasing in size over 1 to 2 years, which is varying from 3 to $10 \mathrm{~cm}$ [3]. SS could easily be confused with benign tumors in the early stage, as the gradual increase of tumor size, which shows the same symptoms as oral squamous cell carcinoma.

For tumors that have recurred multiple times or diameter $>5 \mathrm{~cm}$, the growth rate tends to accelerate and may cause an emergency. In this case, the patient's tumor rapidly increased with surface bleeding after admitted to our hospital. Moreover, he even suffered from severe dyspnea due to the tumor and clot blocking the airway. According to the postoperative tumor anatomy, the central part of the tumor showed liquefaction necrosis with some dark brown liquid (Fig. 7), which may infer to be related to the cause of dyspnea. Therefore, physicians should always pay more attention to the patient's breathing and oxygen saturation for patients with tumor nearing the tongue or soft palate, especially for those with rapid growth or surface bleeding. Preventive tracheostomy may be considered to prevent suffocation.

\section{Diagnosis and differential diagnosis}

In our case, the patient was previously diagnosed as fibroadenoma because of the rarity of SS and lack of typical 

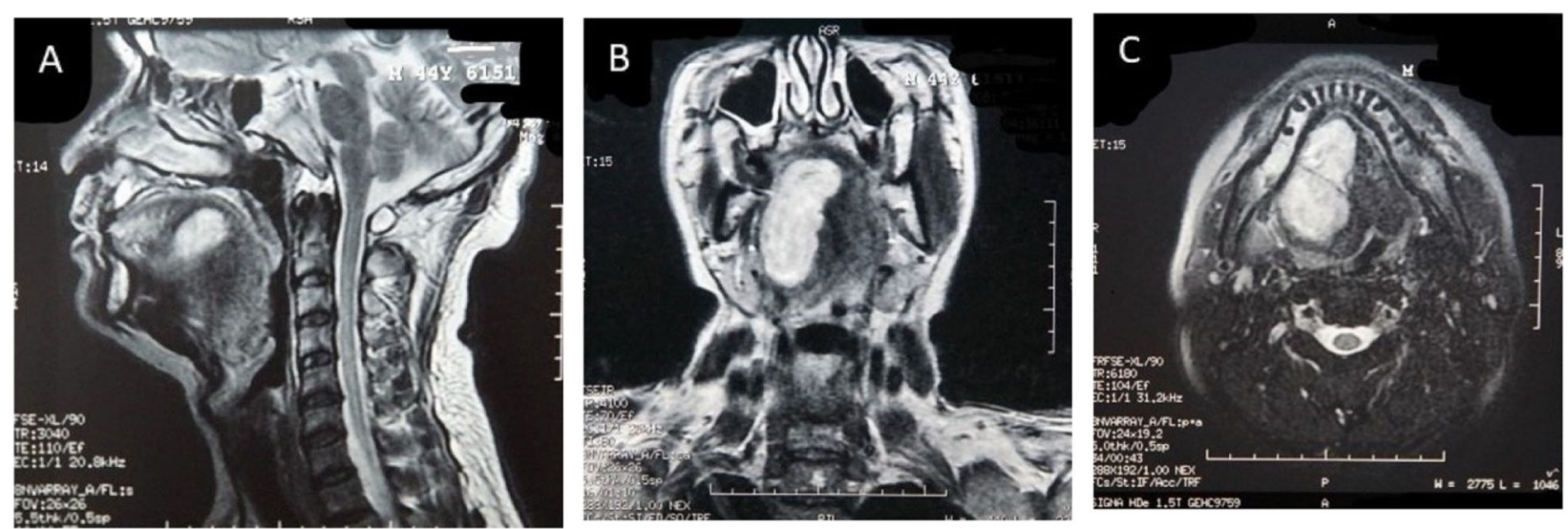

Fig. 2 CT examination of different sections of the patient. a-c There is a clear mass on the right root of tongue and floor of mouth with scattered calcification and liquefaction necrosis

clinical and imaging manifestations. Although Pantomography, CT, MRI, and PET-CT can be used as diagnostic tools, smaller SSs often show similar imaging features to benign tumors on CT and MR imaging $[16,17]$. Despite the lack of specific imaging findings, CT and MRI are still useful for determining the location of the primary tumor, adjacent tissue infiltration, and metastasis.

Synovial sarcoma can be classified into three major histopathological subtypes: 1) monophasic SS containing uniform spindle cells or epithelial cells, 2) biphasic SS composed of epithelial cells arranged into glandular structures with spindle cells arranged into fascicles, 3) poorly differentiated SS characterized by the presence of spindle and/or round blue cells [18]. In histology, synovial sarcoma needs to be differentiated from metastatic adenocarcinoma, malignant fibrous histiocytoma (MFH) and fibrosarcoma. However, due to the diversity of morphological manifestations, the pathological diagnoses of atypical cases are very difficult [19].

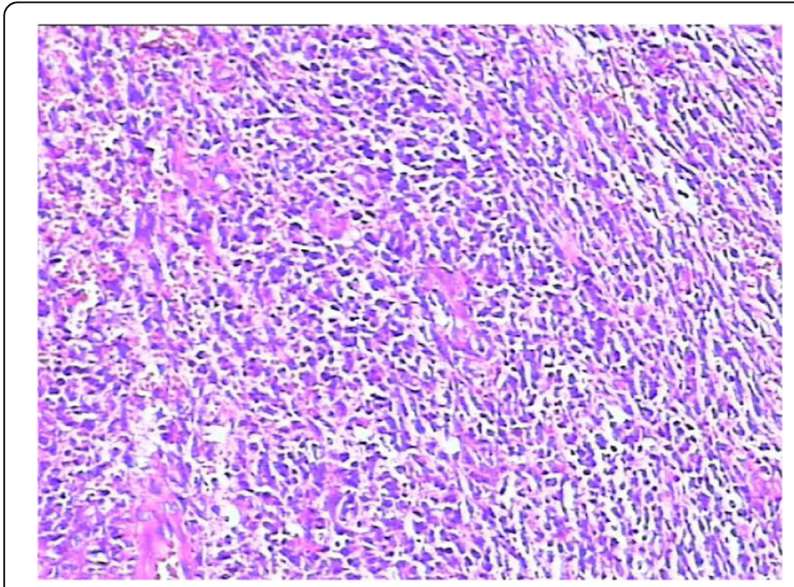

Fig. 3 The hematoxylin and eosin (HE)-stained section revealed the tumor composed of spindle cells with a higher proportion of nuclei. HE. $\times 20$
The other cause of misdiagnosis might be the previous immunohistochemistry lacked some key immune marker detection such as EMA, CD99, TLE-1, and Bcl-2, etc. So far, there is no single immunological marker specific to synovial sarcoma has been found. SS shows positive of TLE-1, AE1 / AE3, EMA, CK7, CK19, Vimentin, Bcl-2, CD99, and S-100 while generally shows negative express of CD34, CD31, actin (HHF-35) or Myoglobin [20, 21]. Some studies reported that TLE-1 expression, a sensitive and specific marker for SS, could be as high as $90 \%$, and Bcl-2 expression rate was 93\%, CD99 was $73 \%$, While S100 was locally expressed in $21 \%$ of cases [22-24].

For some cases with atypical histological morphology or confusing results of immunohistochemistry, detection

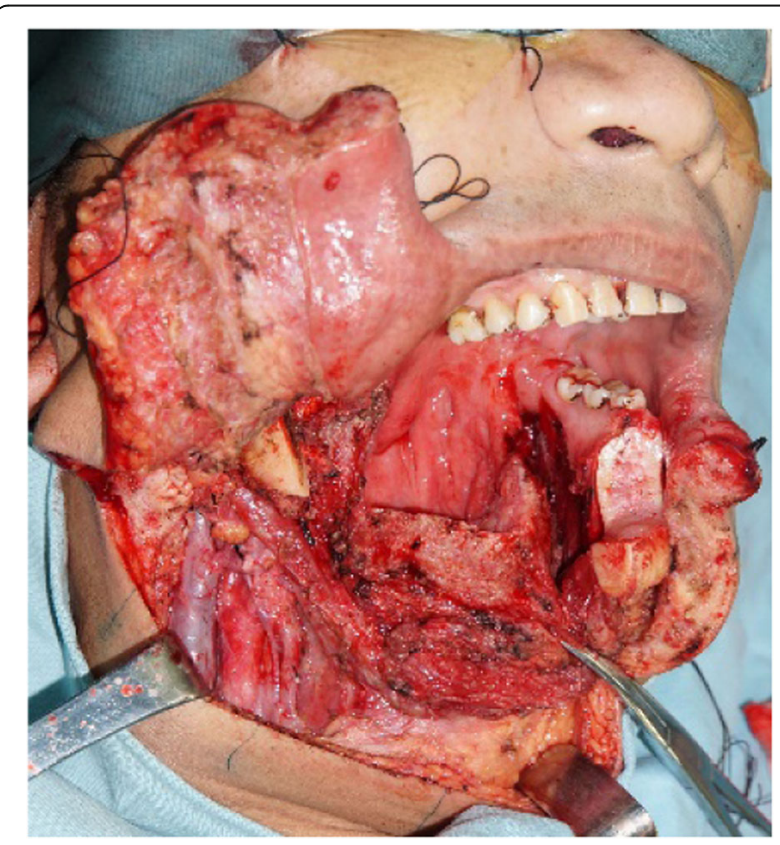

Fig. 4 Resection the total tongue and right mandibular 


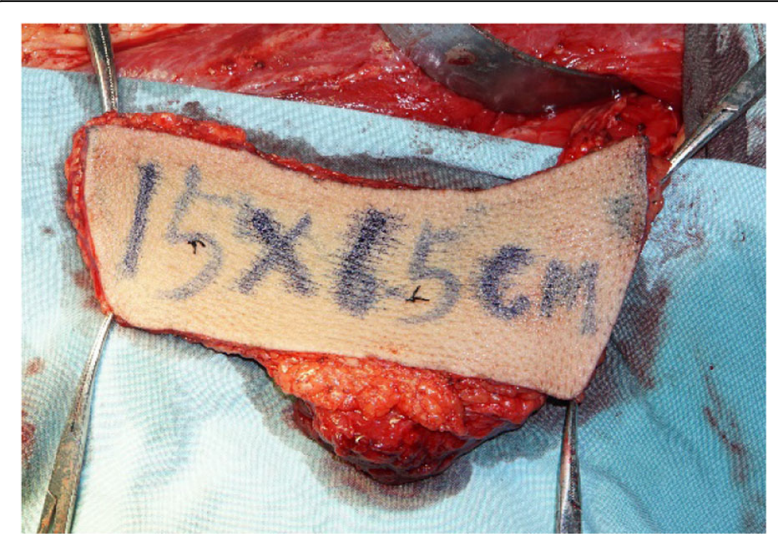

Fig. 5 The left thigh anterolateral musculocutaneous flap with some fascia and muscle

of SYT-SSX fusion gene by molecular biology or cytogenetic technology can be used to help diagnose with synovial sarcoma [20]. It was said that nearly 90 to $95 \%$ of SS demonstrated specific $\mathrm{t}(\mathrm{x} ; 18)$ (p11.2-q11.2) chromosomal translocation that forms the fusion gene SYT-SSX [9-11]. The detection rate of FISH was about $80 \%$ and RT-PCR was $83.8 \%$, and the combined detection rate of both was $92.9 \%$ [25].

\section{Treatment and prognosis}

The optimal approach to the treatment of SS is still unclear and there is no standard treatment protocol [6]. It is recommended wide-local excision [20], and adjuvant radiation with or without chemotherapy [9]. At present, for synovial sarcoma in head and neck, simple surgical treatment can be recommended for smaller and superficial lesions; but for the larger and deeper SS, surgery and radiotherapy combined treatment can be considered since it is difficult to perform completely extended resection as many nerve and vessels involved in the head

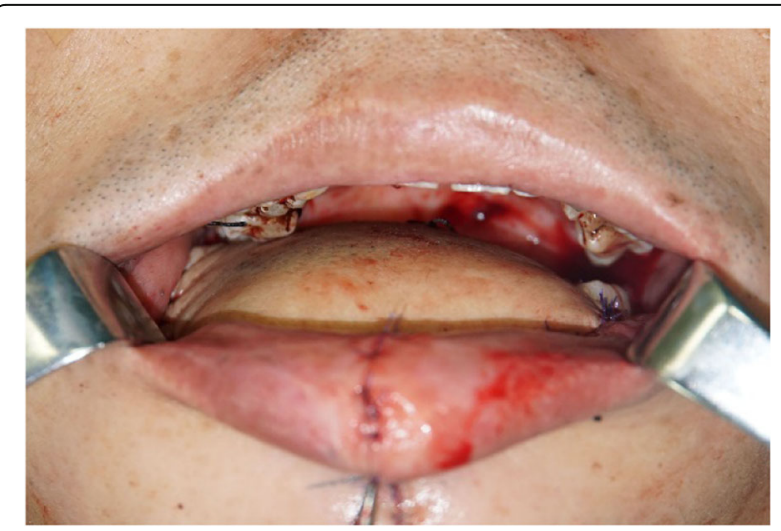

Fig. 6 Instant reconstruction of the floor of mouth defect with left thigh anterolateral musculocutaneous flap

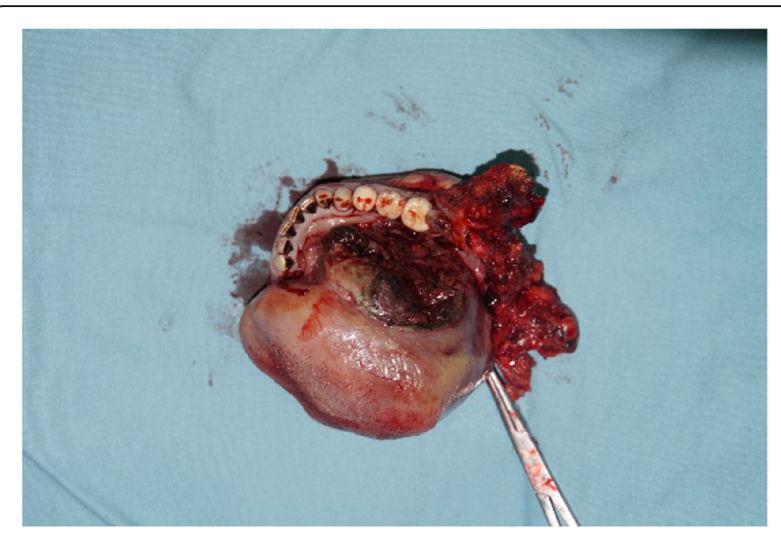

Fig. 7 The central part of tumor that was removed showed liquefaction necrosis with some dark brown liquid

and neck region. The defects after resection can be repaired with flap transfer [26].

In our case, chemotherapy was not performed. The importance of chemotherapy has not been widely acknowledged due to the lack of evidence that chemotherapy was associated with improved overall survival. According to the Yang's report [27], after observation of 21 patients with synovial sarcoma of the head and neck, it was found that postoperative chemotherapy slightly prolonged the time for the occurrence of distant metastasis but showed no significant difference for the overall survival rate or local recurrence. On the other hand, radiotherapy for oral tumors sometimes leads to adverse effects such as radiation mucositis, ulceration, and osteoradionecrosis [1]. According to Zhou's report [28], for soft tissue sarcoma of the maxillofacial region, the five-year survival rate of those who underwent preventive cervical lymph node dissection was higher than those who did not undergo cervical lymph node dissection.

The prognosis of synovial sarcoma is often associated with tumor location, size, patients' age, surgical procedure, degree of differentiation [29]. Generally believed that: 1) age $<60$ years old, 2) total tumor dimension $<5 \mathrm{~cm}, 3$ ) extensive calcification, 4) appropriate surgical resection, 5) a high degree of tumor differentiation, extensive hemorrhagic necrosis and high mitosis index, 6) tumor without distant metastasis, will lead to good prognosis [30-32]. However, due to the insidious onset, patients see doctors always because of discomfort or dysfunction with large tumors, and the curative effect is often poor, the recurrence or metastasis generally occurs in the first two years after the initial treatment [1]. The recurrence rate is $20.8 \%$ and the metastatic rate is $29.2 \%$, the most common metastatic sites are lung, followed by local lymph nodes and bone [1, 30-32]. The three-year survival rate is about 50\% [33].

In this case, as the rarity SS tumor, the patient was misdiagnosed at the local hospital. Immunohistological analysis might be the most important tool to confirm 
the diagnosis of SS. Surgical treatment focus on primary tumor resection without chemotherapy might be considered according to the patient's situation.

\section{Abbreviations}

CT: Computed tomography; EMA: Epithelial membrane antigen; HE: The hematoxylin and eosin; MFH: Malignant fibrous histiocytoma; PETCT: Positron Emission Tomography-Computed Tomography; RT-PCR: Reverse Transcription-Polymerase Chain Reaction; SS: Synovial sarcoma

\section{Acknowledgements}

The authors would like to thank Branden Wang for constructive writing and editing support of the manuscript.

\section{Authors' contributions}

KW performed biopsy for diagnosis. FZ collected data and patient history. YW analyzed the patient data and wrote the article with KW. All authors read and approved the final manuscript.

\section{Funding}

Not applicable.

\section{Availability of data and materials}

The complete data and materials described in the case report are not publicly available due anonymity purposes but are available from the Department of Oral and Maxillofacial Surgery, The Second Xiangya Hospital of Central South University on reasonable request.

\section{Ethics approval and consent to participate}

Authors declare that the need of ethic approval was waived due to the anonymous nature of the report. Local ethics committee ruled that no formal ethics approval was required in this particular case.

\section{Consent for publication}

The patient provided written consent to publish this case report.

\section{Competing interests}

The authors declare that they have no competing interests with regards to authorship and/or publication of this paper.

Received: 26 August 2019 Accepted: 18 November 2019 Published online: 06 January 2020

\section{References}

1. Meer S, Coleman H, Altini M. Oral synovial sarcoma: a report of 2 cases and a review of the literature. Oral Surg Oral Med Oral Pathol Oral Radiol Endod. 2003:96(3):306-15.

2. Grayson W, Nayler SJ, Jena GP. Synovial sarcoma of the parotid gland. A case report with clinicopathological analysis and review of the literature. S Afr J Surg. 1998;36(1):32-4 discussion 34-5

3. Sravya T, Sivaranjani Y, Bhat V, Rao G. Primary biphasic synovial sarcoma of gingiva: Report of a rare case. J Oral Maxillofac Pathol. 2014;18(1):77.

4. Mahesh KTS, Ponnuswamy IA, David MP, Shivhare P, Puttaranganayak MI, Sinha P. Synovial sarcoma of the Buccal mucosa: a rare case report. Case Rep Dent. 2013;2013:1-5

5. Amble FR, Olsen KD, Nascimento AG, Foote RL. Head and neck synovial cell sarcoma. Otolaryngol Head Neck Surg. 1992;107(5):631-7.

6. Harb WJ, Luna MA, Patel SR, Ballo MT, Roberts DB, Sturgis EM. Survival in patients with synovial sarcoma of the head and neck: association with tumor location, size, and extension. Head Neck. 2007:29(8):731-40.

7. Bukawa $\mathrm{H}$, et al. Monophasic epithelial synovial sarcoma arising in the temporomandibular joint. Int J Oral Maxillofac Surg. 2007;36(8):762-5.

8. Zaidi S, Arafah M. Poorly differentiated monophasic synovial sarcoma of the mediastinum. Indian J Pathol Microbiol. 2011;54(2):384.

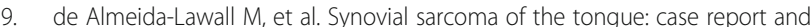
review of the literature. J Oral Maxillofac Surg. 2009;67(4):914-20.

10. Tao Q, Qiao B, Wang Y, Hu F. Diagnosis and treatment of primary synovial cell sarcoma that occurred in the left mandible body: a case report and literature review. Oral Surg Oral Med Oral Pathol Oral Radiol Endod. 2011; $111(2): \mathrm{e} 12-20$
11. Wang H, Zhang J, He X, Niu Y. Synovial sarcoma in the Oral and maxillofacial region: report of 4 cases and review of the literature. J Oral Maxillofac Surg. 2008;66(1):161-7.

12. Pretto D, Barco R, Rivera J, Neel N, Gustavson MD, Eid JE. The synovial sarcoma translocation protein SYT-SSX2 recruits $\beta$-catenin to the nucleus and associates with it in an active complex. Oncogene. 2006;25(26):3661-9.

13. Saito T, Nagai M, Ladanyi M. SYT-SSX1 and SYT-SSX2 interfere with repression of E-cadherin by snail and slug: a potential mechanism for aberrant Mesenchymal to epithelial transition in human synovial sarcoma. Cancer Res. 2006;66(14):6919-27.

14. Tateishi U, Hasegawa T, Beppu Y, et al. Synovial sarcoma of the soft tissues: prognostic significance of imaging features. J Comput Assist Tomogr. 2004; 28(1):140-8

15. Kransdorf MJ. Malignant soft-tissue tumors in a large referral population: distribution of diagnoses by age, sex, and location. Am J Roentgenol. 1995; 164(1):129-34

16. Rangheard AS, Vanel D, Viala J, et al. Synovial sarcomas of the head and neck: CT and MR imaging findings of eight patients. Am J Neuroradiol. 2001;22(5):851-7.

17. Hirsch RJ, et al. Synovial sarcomas of the head and neck: MR findings. Am J Roentgenol. 1997;169(4):1185-8.

18. Jayasooriya PR, Madawalagamage LN, Mendis BRRN, Lombardi T. Diagnostic approach to synovial sarcoma of the head and neck illustrated by two cases arising in the face and Oral cavity. Dermatopathology. 2016:3(1):13-22.

19. Spillane AJ, A'Hern R, Judson IR, Fisher C, Thomas JM. Synovial sarcoma: a Clinicopathologic, staging, and prognostic assessment. J Clin Oncol. 2000; 18(22):3794-803.

20. Vig T, Thomas M, Pai R, et al. Primary Synovial Sarcoma arising from gingivo-buccal sulcus harbouring SS18-SSX2 positive fusion transcript: The 1st reported case in English literature. J Stomatol Oral Maxillofac Surg. 2018; 119(3):220-3.

21. Basile LE, Hoch B, Dillon JK. Synovial sarcoma of the tongue: report of a case. J Oral Maxillofac Surg. 2016:74(1):95-103.

22. Jagdis A, Rubin BP, Tubbs RR, Pacheco M, Nielsen TO. Prospective evaluation of TLE1 as a diagnostic Immunohistochemical marker in synovial sarcoma. Am J Surg Pathol. 2009;33(12):1743-51.

23. Kosemehmetoglu K, Vrana JA, Folpe AL. TLE1 expression is not specific for synovial sarcoma: a whole section study of 163 soft tissue and bone neoplasms. Mod Pathol. 2009;22(7):872-8.

24. Villaroel-Salinas J, Campos-Martinez J, Ortiz-Hidalgo C. Synovial sarcoma of the tongue confirmed by molecular detection of the SYT-SSX2 fusion gene transcript. Int J Surg Pathol. 2012;20(4):386-9.

25. Kanemitsu S, Hisaoka M, Shimajiri S, Matsuyama A, Hashimoto H. Molecular detection of SS18-SSX fusion gene transcripts by CRNA in situ hybridization in synovial sarcoma using formalin-fixed, paraffin-embedded tumor tissue specimens. Diagn Mol Pathol. 2007:16(1):9-17.

26. Mettman A, Myers LL, Carrick K. Synovial sarcoma of the cheek. Ear Nose Throat J. 2009:88(10):1150-2.

27. Yang Y. Diagnosis and treatment of soft tissue sarcoma in China. Chin Clin Oncol. 2010;37(24):1385-9.

28. Zhou Z. Surgical treatment of soft tissue sarcoma in oral and maxillofacial region. J Oral Maxillofac Surg. 1991;2:9-13.

29. Wushou A, Miao X-C. Tumor size predicts prognosis of head and neck synovial cell sarcoma. Oncol Lett. 2015;9(1):381-6.

30. Bukachevsky RP, Pincus RL, Shechtman FG, Sarti E, Chodosh P. Synovial sarcoma of the head and neck. Head Neck. 1992;14(1):44-8.

31. Pai S, Chinoy RF, Pradhan SA, D'cruz AK, Kane SV, Yadav JN. Head and neck synovial sarcomas. J Surg Oncol. 1993;54(2):82-6.

32. Varela-Duran J, Enzinger FM. Calcifying synovial sarcoma. Cancer. 1982:50(2): 345-52.

33. Ameerally P, Sira S, Barrett A, Hollows P. Synovial sarcoma of the hard palate. Br J Oral Maxillofac Surg. 2004;42(3):261-3.

\section{Publisher's Note}

Springer Nature remains neutral with regard to jurisdictional claims in published maps and institutional affiliations. 